\title{
A pathogen mimetic molecule induces highly amplified synergistic immune activation to vaccination via activation of multiple signaling pathways
}

Fnu Naorem Nihesh, ${ }^{\ddagger[a]}$ Saikat Manna, ${ }^{\ddagger[a]}$ Bradley Studnitzer ${ }^{[a]}$, Jingjing Shen ${ }^{[a]}$, Aaron P. EsserKahn.*[a]

[a] N. Nihesh, Dr. S. Manna, B. Studnitzer, Dr. J. Shen, Prof. Dr. A. P. Esser-Kahn

Pritzker School of Molecular Engineering

University of Chicago

5801 S Ellis Ave, Chicago, IL 60637

E-mail: aesserkahn@uchicago.edu

$\ddagger$ F. N. N. and S. M. contributed equally

Abstract: Adjuvants are immunostimulants that enhance the efficacy of vaccines. Unfortunately, often times commonly used adjuvants like alum fail to generate adequate protective responses. Therefore, adjuvants which target pattern recognition receptors (PRRs) in immune cells have been investigated as an alternative approach. However, such adjuvants, like MPLA and CpG activate only a single immune receptor, not fully mimicking the mechanisms of pathogen recognition. To address these challenges, here we developed a molecule that simultaneously activates multiple classes of receptors (TLR, NOD and NLRP) similar to natural pathogen recognition by the immune system. Our in-vitro and in-vivo studies indicated that this molecule induced highly amplified synergistic co-activation of multiple immune signaling pathways, and enhanced $\mathrm{T}$ cell responses to vaccination. Thus, these pathogen mimetic molecules may hold promise for applications in enhancing immunogenicity of subunit vaccines and immunotherapies.

\section{Introduction}

Adjuvants are immunostimulants that enhance the immunogenicity of an antigen in vaccine formulations. Various adjuvants are employed in vaccines to induce robust responses against protein antigens and generate effective protection. However, commonly employed aluminum salt adjuvants predominantly induce humoral responses and do not generate cellular responses 
needed for protection against some infections ${ }^{[1-4]}$ Thereby, pathogen associated molecular patterns (PAMPs) that are recognized by pattern recognition receptors (PRR) in innate immune cells are being investigated for the development of vaccine adjuvants. ${ }^{[5,6]}$ Recent studies on cellular signaling pathways in innate immune cells have demonstrated that PRRs work in concert and that activation of multiple signaling pathways is often critical for efficacious vaccines. $^{[7-9]}$ However, such immunological understanding has yet to be translated to the molecular design of vaccine adjuvants. To date, FDA approved PRR agonist adjuvants, MPLA (TLR4 agonist) and CpG (TLR9 agonist) activate only a single PRR. To explore the hypothesis of multi-PRR activation, we recently demonstrated the development of multi-toll-like receptor (TLR) agonist adjuvants by covalently linking 3 TLR agonists to elicit cellular co-activation of three TLRs and generate tailored immune responses..$^{[10-12]}$

To expand beyond TLRs and capitalize on recent studies showing the critical importance of coactivation of other classes of PRRs for pathogen recognition, we have designed a novel multiPRR agonist. For this design, apart from TLR receptor activation, we targeted NOD2 and NLRP3 receptors based on recent studies which show these innate immune pathways are amplified in response to TLR signaling. For instance, a recent study by Mellman and coworkers demonstrated that initial TLR signaling by bacterial ligands leads to overexpression of endolysosomal peptide transporters and generation of endosomal membrane tubules that enhance NOD2 signaling in innate immune cells. ${ }^{[13]}$ Furthermore, studies on NLRP3 inflammasome activation in innate immune cells demonstrated that initial TLR signaling leads to upregulation of pro-caspase-I, pro-IL1b and pro-IL18 which are subsequently converted to their active forms in presence of a second stimuli that induces NLRP3 inflammasome activation. ${ }^{[14-18]}$ Inspired by these findings, we generated a new molecule which we conjectured would induce co-activation of TLR2, NOD2 and NLRP3 signaling pathways in innate immune cells. We envisioned that this single molecule would recapitulate the activation of multiple classes of pattern recognition receptors akin to natural pathogen recognition and elicit robust immune activation. Designs like these might form the basis for potent immuno-therapeutics and vaccine adjuvants. 


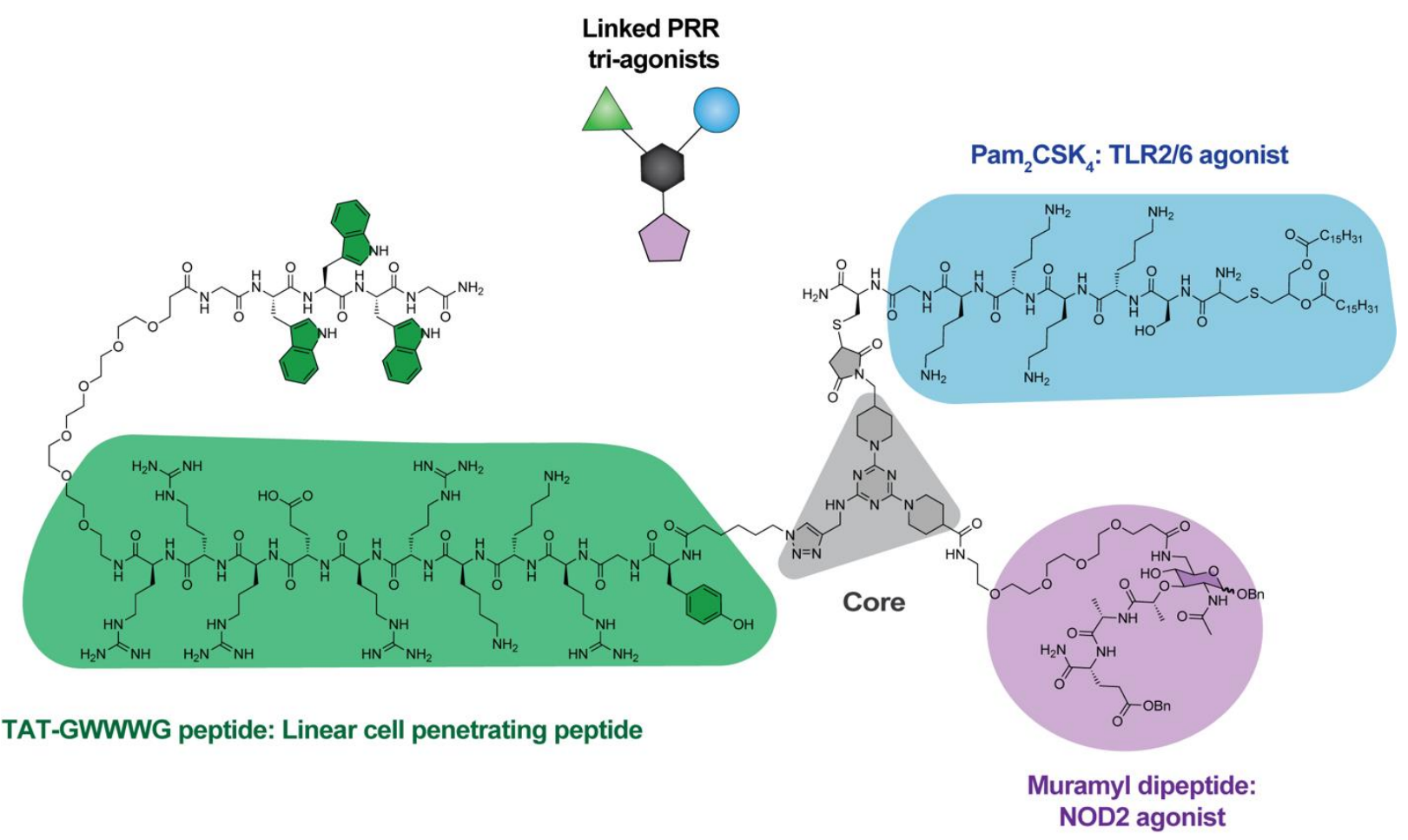

Figure 1. Structure of multi-PRR tri-agonist consisting of Muramyl dipeptide (NOD2 agonist), $\mathrm{Pam}_{2} \mathrm{CSK}_{4}$ (TLR2 agonist) and TAT-GWWWG (cell penetrating peptide).

\section{Results and Discussion}

Design and Synthesis. To generate a trimeric immune agonist adjuvant that activated TLR2, NOD2 and NLRP3 signaling pathways, we employed $\mathrm{Pam}_{2} \mathrm{CSK}_{4}$ (TRL2/6 agonist), Muramyl dipeptide (MDP, NOD2 agonist), and TAT-GWWWG peptide (cell penetrating peptide, NLRP3 activator). The TAT-GWWWG peptide by itself is not immunogenic but serves a dual purpose. The compound activates NLRP3 when administered following a TLR stimulation (TLR2/6 in this case) thereby inducing inflammasome activation. ${ }^{[19]}$ Additionally, to our surprise, we observed that when chemically conjugated to MDP, TAT-GWWWG enhanced NOD2 activation (Figure S2). We conjecture that this enhancement is the result of the endo-osmolytic property of the peptide increasing cytosolic delivery of MDP. ${ }^{[20]}$

To generate the new molecule, we adapted the synthetic scheme used for our TLR tri-agonist system, using a triazine core and sequential orthogonal conjugation chemistry (Scheme 1). We synthesized core (1) with three orthogonal conjugation handles, an NHS ester, an alkyne, and a protected maleimide (Scheme S2). ${ }^{\left[{ }^{[1]}\right.}$ An amine-functionalized MDP (MDP-PEG $4-\mathrm{NH}_{2}$ (a)) was synthesized following previously reported synthesis (Scheme S1). ${ }^{[22]} \mathrm{Pam}_{2} \mathrm{CSK}_{4} \mathrm{GC}$ (b) (thiol 
modified) and GWWWG-Peg 6 -TAT-hex- $\mathrm{N}_{3}$ (c) peptide (azide modified) were synthesized using microwave assisted solid phase peptide synthesis. MDP-PEG $-\mathrm{NH}_{2}$ (a) was conjugated to core (1) using NHS chemistry. Briefly, MDP-PEG $-\mathrm{NH}_{2}$ (a), core (1) and TEA were dissolved in DMF and the mixture was heated at $50{ }^{\circ} \mathrm{C}$ for $3 \mathrm{~h}$ to give core_MDP (2). core_MDP (2) was then dissolved in anhydrous DMSO and the mixture was heated to $110{ }^{\circ} \mathrm{C}$ for $5 \mathrm{~h}$ to reveal the protected maleimide. Once the furan deprotection was complete, $\mathrm{Pam}_{2} \mathrm{CSK}_{4} \mathrm{GC}$ (b) was added to the mixture and stirred at room temperature for $1 \mathrm{~h}$ to give $\mathrm{Pam}_{2} \mathrm{CSK}_{4}$ core_MDP (3). This was followed by conjugation of GWWWG-Peg6-TAT-hex- $\mathrm{N}_{3}$ (c) to $\mathrm{Pam}_{2} \mathrm{CSK}_{4}$ _core_MDP (3) via $\mathrm{Cu}(\mathrm{I})$ catalyzed Huisgen cycloaddition chemistry to afford the TAT-GWWWG_Pam ${ }_{2} \mathrm{CSK}_{4}$ MDP tri-agonist molecule (4). The tri-agonist was purified by HPLC (Figure S3) and characterized by MADLI-TOF (Figure S4). During the convergent synthesis, all of the dimeric agonist compounds, $\mathrm{PAM}_{2} \mathrm{CSK}_{4}$ MDP (3) (Scheme S3), PAM $\mathrm{CSK}_{4}$ TAT-GWWWG (7) (Scheme S5) and MDP_TAT-GWWWG (8) (Scheme S4) were synthesized to measure the contribution of each component to the immune response.

Tri-agoinst generates robust immune co-activation. To examine how thevocalnet attachment of the three agnoists affected theimmune response, we analyzed the cytokine profile elicited by the multi-PRR stimulation of murine bone marrow-derived dendritic cells (BMDCs) in vitro. BMDCs were stimulated with the linked or unlinked PRR agonsits (100 nM) for 6h. Parallel studies were performed using equivalent quantities of linked di-agonists (MDP_PAM $\mathrm{CSK}_{4}$, $\mathrm{PAM}_{2} \mathrm{CSK}_{4}$ TAT-GWWWG, TAT-GWWWG_ MDP) and comparing them against the corresponding single agonists $(100 \mathrm{nM})$, for the same duration. Analysis of levels of cytokine secreted by agonist stimulation indicated that the linked tri-agonist combinations elicited at least $150 \%$ higher IL- 6 and TNF-a production compared to the various di-agonist combinations and $400 \%$ higher than the single agonists (Figure 2D, 2E) implying that cellular co-activation with all three ligands was responsible for the enhanced immune response. Most notably, compared to an equivalent mixture of unlinked agonists, linking the three ligands boosted the synergistic IL-6 and TNF-a secretion by at least $160 \%$ (Figure 2D, 2E). These results provided evidence for our hypothesis that synergistic cellular co-activation by localization of multiple PRRs enhanced immune activation. ${ }^{[23]}$

With these exciting results, we next proceeded to analyze whether the tri-agonist activated all the target PRRs. We analyzed cytokine secretion elicited by the tri-agonist stimulation on wild type BMDCs and NOD2 knockout BMDCs. Intriguingly, the NOD2 knockout BMDCs expressed $65 \%$ lower TNF-a and $71 \%$ lower IL-6 levels compared to the wild type BMDCs implying that a 


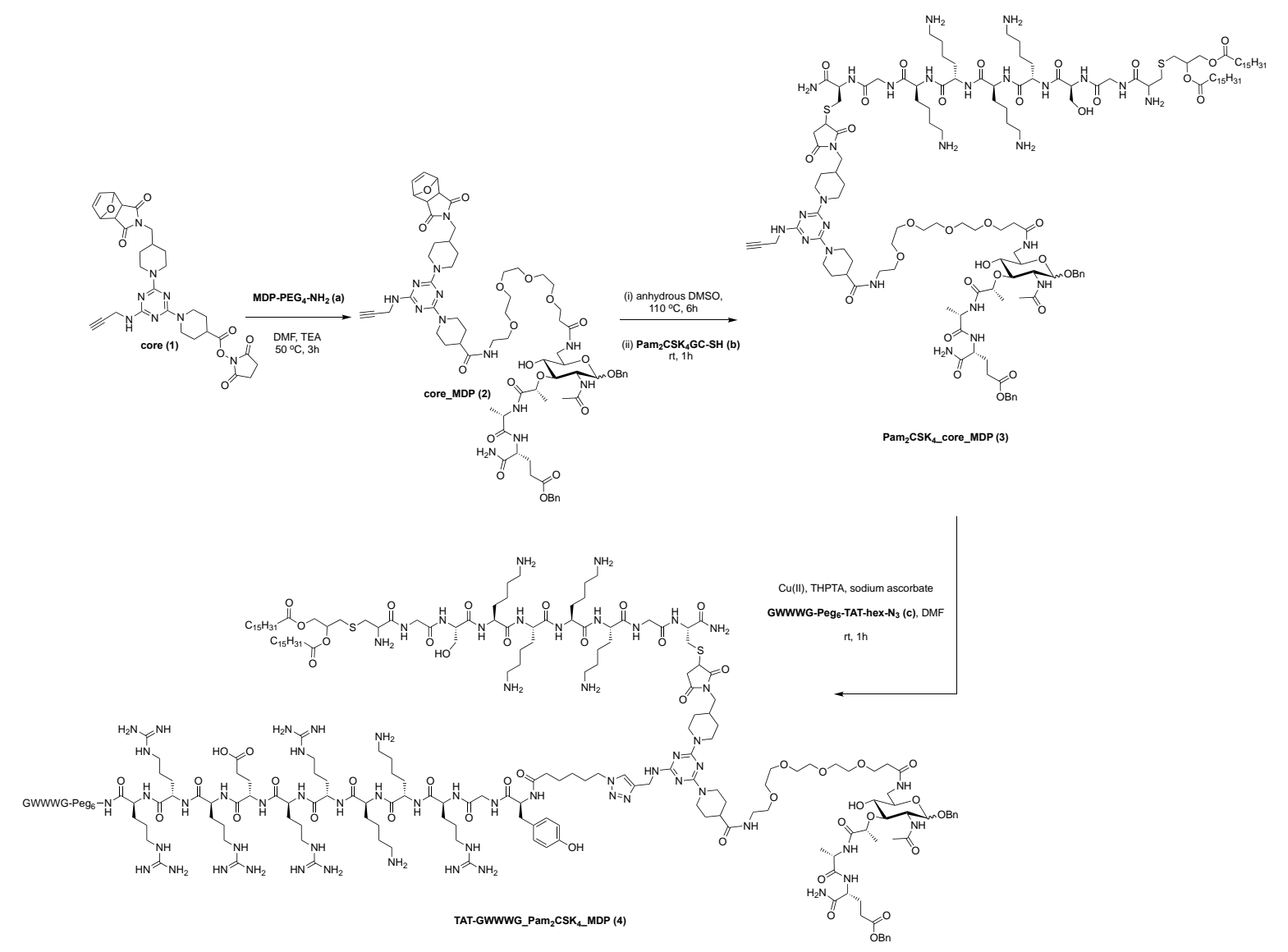

Scheme1: Synthesis of TAT-GWWWG_Pam 2 CSK $_{4}$ MDP (4) tri-agonist

significant enhancement in immune response was a result of the crosstalk between NOD2 and TLR2 receptors (Figure $2 \mathrm{~A}, 2 \mathrm{~B}$ ). In addition to this result, TLR2 knockout BMDCs expressed near background level cytokines indicating that TLR2 signaling initiated the immune activation fitting what has been observed previously about the need for pre-activation via a Toll-like Receptor to then activate NOD2 and NLRP3.

To determine the influence of the linked tri-agonist molecule to elicit NLRP3-infllammasome activation, we analyzed secretion of IL-1b, a common marker of inflammasome activation. ${ }^{[16,18]}$ Stimulation with the linked tri-agonist resulted in $200 \%$ higher levels of IL-1b compared to an equivalent mixture of unlinked agonists (Figure S4). To further explore the inflammasomes involvement with synergistic activity, we performed knockout studies. BMDCs lacking NLRP3 (NLRP3 -/-) elicited lower cytokine responses (TNF-a, IL-6) compared to WT BMDCs (Figure $2 \mathrm{~A}, 2 \mathrm{~B})$, however, such effects might also be the result of reduction in MDP activity observed in NLRP3 -/- cells. To differentiate between direct NLRP3 contribution and secondary effects, we 
performed further studies with WT BMDCs employing a NLRP3 specific inhibitor, MCC-950. Coincubation of WT BMDCs with the tri-agonist and MCC 950 reduced IL-1b activity by $62 \%$ indicating NLRP3 inflammasome activation by the tri-agonist (Figure $2 \mathrm{C}){ }^{[24,25]}$

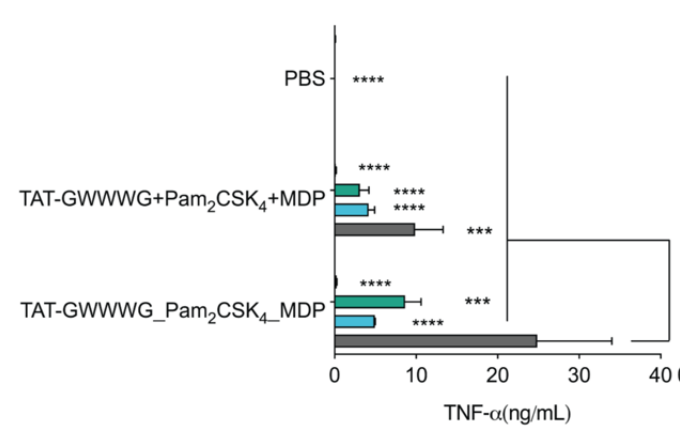

A

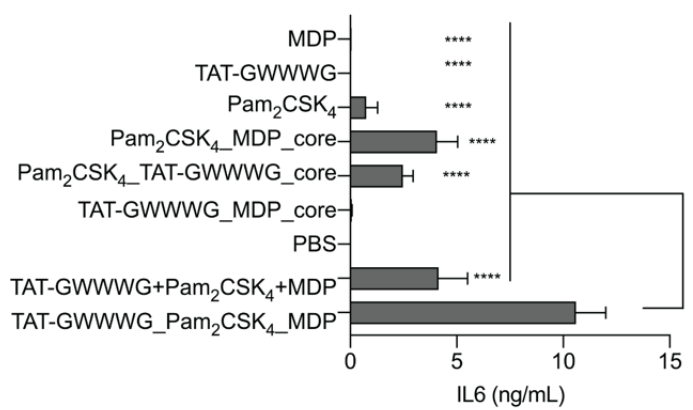

D

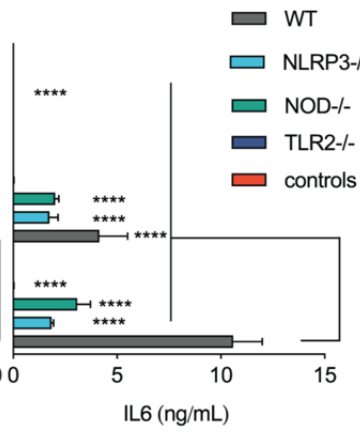

B

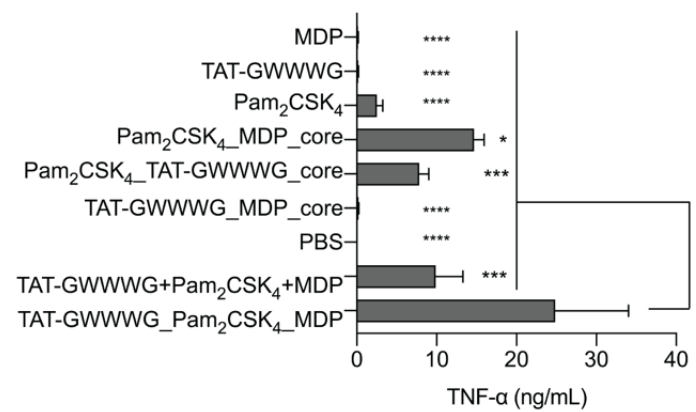

$\mathrm{E}$

Figure 2. In vitro cytokine expression from BMDCs measured by CBA (TNF-a, IL-6) and ELISA (IL-1b). (A), (B): Cells were incubated with linked PRR tri-agonist (100 nM) or a 1:1:1 (molar ratio) mixture of the analogous unlinked PRR agonists (100 nM each) at $37^{\circ} \mathrm{C}$ for $6 \mathrm{~h}$. The cells treated with linked PRR triagonist (TAT-GWWWG_Pam $2 \mathrm{CSK}_{4} \_$MDP) elicited the highest expression of TNF-a and IL-6. The increase in response was strongly dependent on TLR2 activation. (C): Confirming activation of NLRP3. Cells were preincubated with MCC $950(10 \mathrm{uM})$ for $1 \mathrm{~h}$ and then stimulated with linked PRR tri-agonist (TAT-GWWWG_Pam $2 \mathrm{CSK}_{4} \_\mathrm{MDP}, 10 \mathrm{uM}$ ) at $37^{\circ} \mathrm{C}$ for $24 \mathrm{~h}$. Inhibition of NLRP3 via MCC 950 results in loss of IL-1b activity. (D), (E): Linking the three ligands strongly boosted the cytokine production compared to the unlinked mixture, single agonist or various linked di-agonists. Samples were run in triplicate, where, ${ }^{*} p<0.05,{ }^{* * *} p<0.001,{ }^{* * * *} p<0.0001$. Statistical analysis is performed using ANOVA by the Turkey's multiple comparison test 

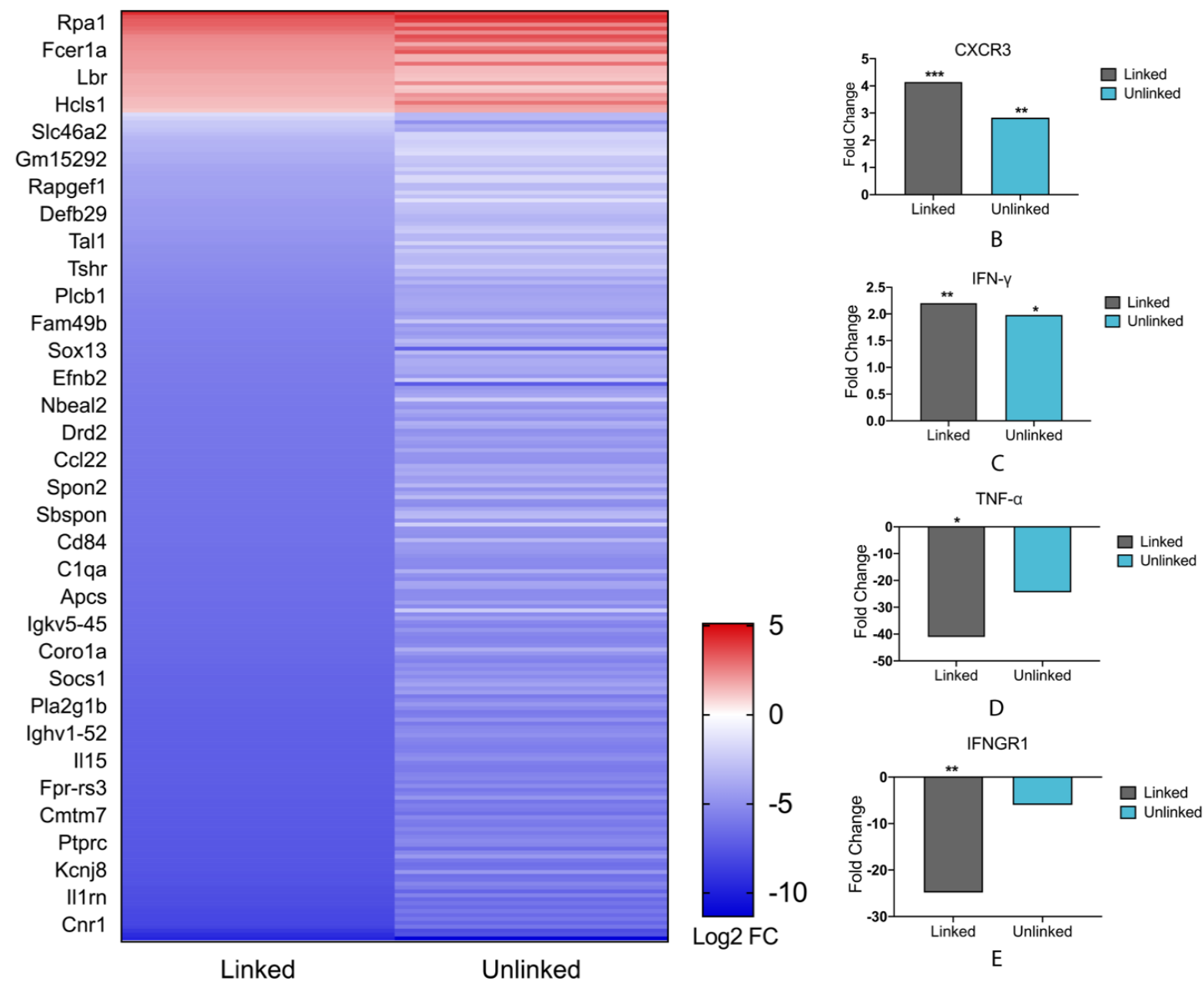

A

Figure 3. BMDC gene expression profile data. (A) Heat map of immune function related genes. Each figure represents the average of three independent experiments. BMDCs were incubated as untreated, or with either the linked or unlinked tri-agonist combination for $6 \mathrm{~h}$ at $37^{\circ} \mathrm{C}$. RNA was then extracted and sequenced on a NextSeq550. The gene expression of the BMDCs in response to unlinked and linked triagonist stimulation was compared to unstimulated BMDCs to determine the differential gene expression profiles. Included in the heatmap are only immune-associated genes with $p$ value $<.05$ for either the linked or unlinked tri-agonists and a 2-fold change in expression. (B), (C), (D), (E): Fold change in gene expression for CXCR3, IFN- $\gamma$, TNF- $\alpha$, IFNGR1 in BMDCs in response to linked and unlinked tri-agonist combination where ${ }^{*} p<0.05,{ }^{* *} p<0.01$, and ${ }^{* * *} p<0.001$. P-values are calculated relative to unstimulated BMDCs. 
The synergistic interaction of the multi-PRR stimulation and knockout indicated that more than a simple interaction between receptors might be occurring. To further analyze how linking these compounds might be affecting their activity, we studied the transcriptional response in BMDCs after stimulation with the unlinked and linked tri-agonist. The RNA of BMDCs was extracted after $6 \mathrm{~h}$ of stimulation with the unlinked and linked tri-agonist, along with unstimulated BMDCs. The differential gene expression for the unlinked and linked tri-agonist was compared to the unstimulated BMDCs to provide insight into the response to this tri-agonist activation. As seen in the heatmap (Figure $3 \mathrm{~A}$ ), we observed a similar trend in responses when the BMDCs were activated with the linked or the unlinked tri-agonist. For both the linked and unlinked triagonists, we see each gene essentially upregulated or downregulated in response to stimulation. Since both the linked and unlinked tri-agonists target the same receptors, they share similar responses. As such, the key differences in expression appear to be the extent and magnitude of this regulation. For example, compared to the unlinked mixture, stimulation with the linked tri-agonist generated higher expression of CXCR3, a key receptor for interferon induced chemo-attractants that helps differentiate naïve $T$ cells into $T_{h} 1$ effector $T$ cells (Fig. 3B). Additionally, we see greater differences in expression for both interferon-gamma (IFN-y) and interferon gamma receptor 1 (IFNGR1) - which dimerizes with IFNGR2 to detect IFN- $\mathrm{\gamma}$, in response to the linked tri-agonist (Figure 3C, 3E). The greater upregulation of IFN- $\gamma$, along with the greater downregulation of IFNGR1, indicates that the tri-agonist is inducing high levels of IFN-y signaling. This can possibly be an effect of enhanced IL-18 secretion due inflammasome activation by the linked tri-agonist. ${ }^{[26]}$ By observing these high expression levels of CXCR3 and IFN- $\mathrm{Y}$, we hypothesize that the linked tri-agonist would induce strong $\mathrm{T}$ cell responses, in particular $T_{h} 1$ polarized responses.

It is worth noting that TNF-a is strongly downreglated in the gene expression, a distinct difference compared to the high TNF-a cytokine levels observed at $6 \mathrm{~h}$ (Figure 3D). This difference highlights the kinetic components of a cellular respose. This difference highlights the kinetic components of a cellular response. These BMDCs are secreting high levels of TNF- $\alpha$ at $6 \mathrm{~h}$, indicating that they are releasing more proinflammatory signals after tri-agonist stimulation, simultaneously, the BMDCs are mediating this proinflammatory response by downregulating their gene expression of TNF- $\alpha$. While it is difficult to conclude that kinetics is the main mechanistic difference between linked and unlinked agonisms with only one transcriptional time point, the strong differences at this single time point do strongly support a difference in the kinetics of transcription. 
Tri-agonist as a potent vaccine adjuvant. With promising in vitro results and the indication of stronger T-cells responses, we proceeded to determine if amplified immune response from the linked tri-agonist would translate to its use as a vaccine adjuvant in vivo. Groups of five mice were

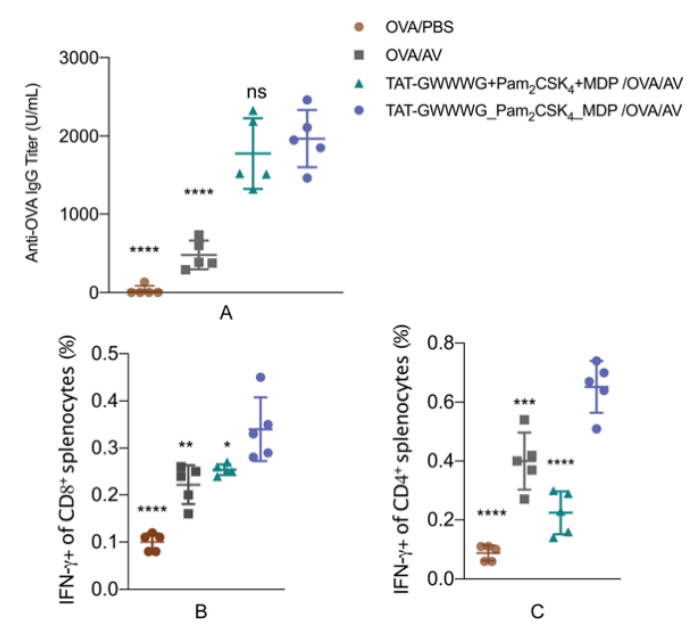

Figure 4. Mice were vaccinated on day 0 with OVA (100 ug) adjuvanted with PBS (vehicle control), or Addavax (25 uL), or 5 nmole each of unconjugated multi-PRR agonist, TAT-GWWWG+Pam ${ }_{2} \mathrm{CSK}_{4}+\mathrm{MDP}$ in Addavax (AV, $25 \mathrm{uL}$ ), or 5 nmole of conjugated agonists, TAT-GWWWG_Pam $2 \mathrm{CSK}_{4}$ _MDP in Addavax (25 uL). Final volume of each formulation was made $50 \mathrm{uL}$ with PBS. Mice were given a vaccine boost on day 14. On day 24, sera, spleens were collected from mice. Antibody titer was measured by ELISA and T cell response was measured by intracellular cytokine staining. Samples were run in quintuplicate, where ns $=$ non-significant, ${ }^{*} p<0.05,{ }^{* *} p<0.01,{ }^{* \star *} p<0.001,{ }^{* \star \star *} p<0.0001$. Statistical analysis is performed between the linked tria-agonist and indicated groups using ANOVA by the Turkey's multiple comparison test.

immunized via IM injection with ovalbumin (OVA, $100 \mathrm{mg}$ ) adjuvanted with linked tri-agonist (5 $\mathrm{nmol}$ ) or equivalent quantities of unlinked agonist mixture. Vaccines were formulated in Addavax (AV), an MF-59 like oil-in-water nano-emulsion. ${ }^{[27]}$ Parallel studies were also performed with unadjuvanted OVA or OVA admixed with Addavax as controls. Mice were boosted in an identical fashion on day 14. On day 24 blood sera were collected to analyze for antibody titer and splenocytes were harvested to analyze for antigen-specific $T$ cell responses (Figure 4B, 4C). Notably the formulation with the linked tri-agonist enhanced IFN- $\gamma$ secreting $\mathrm{CD}^{+}{ }^{+} \mathrm{T}$ cells response by $200 \%$ ( $0.6 \%$ of cells) and IFN- $\gamma$ secreting CD $8^{+}$T cells response by $40 \%(0.35 \%$ of cells) compared to unlinked combination of agonists $(0.2 \%$ and $0.25 \%$ of cells respectively). It also elicited $300 \%$ higher antibody responses compared to OVA/Addavax formulation (Figure 
4A). These results demonstrated that the covalently linked combination of $\mathrm{Pam}_{2} \mathrm{CSK}_{4}, \mathrm{MDP}$, and TAT-GWWWG served as a potent adjuvant in vivo.

These results thus indicate that a single, spatially defined tri-agonist molecule that activates TLR2, NOD2 and NLRP3 receptors in vivo, shows stronger adjuvanticity compared to the unlinked soluble mixture. This strong adjuvant effect can be explained by enhanced cellular coactivation. However, a role of improved pharmacokinetics with the tri-agonist combination cannot be ruled out. For instance, systemic diffusion of MDP and TAT-GWWWG peptide can possibly be significantly restricted via covalent conjugation to the $\mathrm{Pam}_{2} \mathrm{CSK}_{4}$. Nevertheless, taking all the results together, the linked tri-agonist provides a unique immune stimulatory event which has potential to improve cellular responses in vaccine adjuvants.

\section{Conclusion}

In summary, we developed a small-molecule trimeric PRR agonist-based adjuvant inspired by the stimulation pattern of a pathogen. This molecule generated by covalently linking TLR2/6 agonist, NOD2 agonist, and NLRP3 inflammasome activator, stimulates multiple subfamilies of PRRs in a spatially defined manner resulting in an amplified innate immune response in vitro. Moreover, it elicits both stronger humoral and cellular immune responses in vivo. This strategy of taking advantage of synergies amongst different subfamilies of PRRs can help develop vaccines against challenging and emerging diseases by exploiting elements of molecular design to improve immune response.

\section{Acknowledgements}

This research was supported by a grant from NIH (5U01Al124286-05).

Keywords: adjuvant $\bullet$ vaccines $\cdot$ pathogens $\bullet$ immunostimulants $\bullet$ antigen $\cdot$ pathogen associated molecular patterns $\cdot$ pattern recognition receptors

\section{References}

[1] M. Singh, M. Ugozzoli, J. Kazzaz, J. Chesko, E. Soenawan, D. Mannucci, F. Titta, M. Contorni, G. Volpini, G. D. Guidice, D. T. O'Hagan, Vaccine 2006, 24, 1680-1686.

[2] R. A. Seder, A. V. S. Hill, Nature 2000, 406, 793-798.

[3] G. Leroux-Roels, Vaccine 2010, 28, C25-C36.

[4] J. A. Berzofsky, J. D. Ahlers, J. Janik, J. Morris, S. Oh, M. Terabe, I. M. Belyakov, J Clin Invest 2004, 114, 450-462. 
[5] N. W. Palm, R. Medzhitov, Immunological Reviews 2009, 227, 221-233.

[6] R. L. Coffman, A. Sher, R. A. Seder, Immunity 2010, 33, 492-503.

[7] T. Querec, S. Bennouna, S. Alkan, Y. Laouar, K. Gorden, R. Flavell, S. Akira, R. Ahmed, B. Pulendran, J Exp Med 2006, 203, 413-424.

[8] B. Pulendran, Nature Reviews Immunology 2009, 9, 741-747.

[9] V. Pavot, N. Rochereau, J. Rességuier, A. Gutjahr, C. Genin, G. Tiraby, E. Perouzel, T. Lioux, F. Vernejoul, B. Verrier, S. Paul, The Journal of Immunology 2014, 193, 5781-5785.

[10] J. K. Tom, E. Y. Dotsey, H. Y. Wong, L. Stutts, T. Moore, D. H. Davies, P. L. Felgner, A. P. EsserKahn, ACS Cent. Sci. 2015, 1, 439-448.

[11] T. J. Albin, J. K. Tom, S. Manna, A. P. Gilkes, S. A. Stetkevich, B. B. Katz, M. Supnet, J. Felgner, A. Jain, R. Nakajima, A. Jasinskas, A. Zlotnik, E. Pearlman, D. H. Davies, P. L. Felgner, A. M. Burkhardt, A. P. Esser-Kahn, ACS Cent. Sci. 2019, 5, 1137-1145.

[12] A. P. Gilkes, T. J. Albin, S. Manna, M. Supnet, S. Ruiz, J. Tom, A. J. Badten, A. Jain, R. Nakajima, J. Felgner, D. H. Davies, S. A. Stetkevich, A. Zlotnik, E. Pearlman, A. Nalca, P. L. Felgner, A. P. Esser-Kahn, A. M. Burkhardt, J.I. 2020, 204, 611-621.

[13] N. Nakamura, J. R. Lill, Q. Phung, Z. Jiang, C. Bakalarski, A. de Mazière, J. Klumperman, M. Schlatter, L. Delamarre, I. Mellman, Nature 2014, 509, 240-244.

[14] K. V. Swanson, M. Deng, J. P.-Y. Ting, Nature Reviews Immunology 2019, 19, 477-489.

[15] Y. Yang, H. Wang, M. Kouadir, H. Song, F. Shi, Cell Death \& Disease 2019, 10, 1-11.

[16] K. Schroder, J. Tschopp, Cell 2010, 140, 821-832.

[17] M. Lamkanfi, V. M. Dixit, The Journal of Immunology 2011, 187, 597-602.

[18] H. Guo, J. B. Callaway, J. P.-Y. Ting, Nature Medicine 2015, 21, 677-687.

[19] S. Manna, W. J. Howitz, N. J. Oldenhuis, A. C. Eldredge, J. Shen, F. N. Nihesh, M. B. Lodoen, Z. Guan, A. P. Esser-Kahn, ACS Cent. Sci. 2018, 4, 982-995.

[20] P. Lönn, A. D. Kacsinta, X.-S. Cui, A. S. Hamil, M. Kaulich, K. Gogoi, S. F. Dowdy, Scientific Reports 2016, 6, DOI 10.1038/srep32301.

[21] R. Banerjee, N. J. Pace, D. R. Brown, E. Weerapana, J. Am. Chem. Soc. 2013, 135, 2497-2500.

[22] C. L. Grimes, D. K. Podolsky, E. K. O'Shea, Bioorganic \& Medicinal Chemistry Letters 2010, 20, 6061-6063.

[23] B. Lin, B. Dutta, I. D. C. Fraser, Cell Systems 2017, 5, 25-37.e3.

[24] R. C. Coll, J. R. Hill, C. J. Day, A. Zamoshnikova, D. Boucher, N. L. Massey, J. L. Chitty, J. A. Fraser, M. P. Jennings, A. A. B. Robertson, K. Schroder, Nature Chemical Biology 2019, 15, 556-559.

[25] R. C. Coll, A. A. B. Robertson, J. J. Chae, S. C. Higgins, R. Muñoz-Planillo, M. C. Inserra, I. Vetter, L. S. Dungan, B. G. Monks, A. Stutz, D. E. Croker, M. S. Butler, M. Haneklaus, C. E. Sutton, G. Núñez, E. Latz, D. L. Kastner, K. H. G. Mills, S. L. Masters, K. Schroder, M. A. Cooper, L. A. J. O’Neill, Nature Medicine 2015, 21, 248-255.

[26] D. Stober, R. Schirmbeck, J. Reimann, The Journal of Immunology 2001, 167, 957-965.

[27] H. E. Sahly, Expert Review of Vaccines 2010, 9, 1135-1141. 